\title{
Thyroid Carcinoma with NSD3::NUTM1 Fusion: a Case with Thyrocyte Differentiation and Colloid Production
}

\author{
Derek B. Allison ${ }^{1} \cdot$ Justin Rueckert ${ }^{1} \cdot$ Virgilius Cornea $^{1} \cdot$ Cortney Y. Lee ${ }^{2} \cdot J^{\prime}$ Jie Dueber $^{1} \cdot$ Therese Bocklage $^{1}$ (i)
}

Accepted: 1 December 2021 / Published online: 8 January 2022

(c) The Author(s) 2021

\begin{abstract}
In this report, we present a high-grade thyroid carcinoma with an NSD3::NUTM1 fusion detected on expanded next-generation sequencing testing. Nuclear protein of the testis (NUT) carcinomas comprise high-grade, aggressive tumors characterized by rearrangements of the NUTM1 gene with various partner genes, most commonly the bromodomain protein genes BRD4 and BRD3. Approximately 10\% of NUT carcinomas contain an NSD3::NUTM1 fusion. NUT carcinomas manifest as poorly differentiated or undifferentiated squamous carcinomas, and 33\% show areas of mature squamous differentiation. Only exceptionally have NUT carcinomas shown histology discordant from poorly differentiated/undifferentiated squamous carcinoma, and a thyroid NUT carcinoma with histologic thyrocyte differentiation has not been described to date. Our patient's tumor exhibited mixed cytologic features suggestive of squamoid cells or papillary thyroid carcinoma cells. Overt squamous differentiation was absent, and the tumor produced colloid in poorly formed follicles. Immunophenotypically, the carcinoma was consistent with thyrocyte differentiation with expression of monoclonal PAX8, TTF1, and thyroglobulin (the last predominantly in extracellular colloid). There was zero to $<2 \%$ reactivity for proteins typically diffusely expressed in NUT carcinoma: p40, p63, and cytokeratins 5/6. NUT protein expression was equivocal, but fluorescence in situ hybridization confirmed a NUTM1 rearrangement. This exceptional case suggests that NUTM1 fusions may occur in an unknown number of aggressive thyroid carcinomas, possibly with distinctive histologic features but with thyrocyte differentiation. Recognition of this entity potentially has significant prognostic implications. Moreover, thyroid carcinomas with NUTMI fusions may be amenable to treatment with NUT carcinoma-targeted therapy such as a bromodomain and extraterminal domain protein small molecular inhibitor (BETi).
\end{abstract}

Keywords Thyroid carcinoma $\cdot$ NUT carcinoma $\cdot$ NSD3 $\cdot$ NSD3::NUTM1 $\cdot$ BRD4 $\cdot$ NUTM1

\section{Introduction}

Nuclear protein of the testis (NUT) carcinoma (previously termed NUT midline carcinoma) is an aggressive carcinoma that was first described in single case reports beginning in 1991 with the description of an aggressive thymic carcinoma showing a gene translocation, $\mathrm{t}(15 ; 19)(\mathrm{q} 15 ; \mathrm{p} 13)$ [1]. Subsequently, a joint Japanese and American team

Therese Bocklage

therese.bocklage@uky.edu

1 Department of Pathology and Laboratory Medicine, University of Kentucky College of Medicine, Lexington, KY, USA

2 Department of Surgery, Division of Endocrine Surgery, University of Kentucky College of Medicine, Lexington, KY, USA characterized NUT carcinoma as a distinct entity in 2003 [2]. To date, approximately 200 cases of NUT carcinoma have been reported [3]. The typical NUT carcinoma harbors $B R D 4, B R D 3, N S D 3$, or $Z 4$ bromodomain complex (BDC) protein genes as fusion partners to NUTMI and manifests histologically as an undifferentiated to poorly differentiated carcinoma [4]. Keratinization is detected in approximately one-third of cases [4]. Thus, NUT carcinoma has been considered a variant of high-grade squamous carcinoma.

The initial patients reported to have NUT carcinoma were usually children to young adults with a midline tumor. However, the name, "NUT midline carcinoma," was replaced by "NUT carcinoma," as more patients with non-midline tumors were identified. The additional involved reported sites include the lung, salivary glands, pancreas, kidney, adrenal gland, and bladder [4, 5]. Recently, three cases of NUT carcinoma were described as arising in the thyroid 
gland [6-8]. Two of the three cases showed characteristic histologic features of NUT carcinoma, of which one also expressed PAX8 in addition to diffuse $\mathrm{p} 63$ by immunohistochemistry $[6,7]$. The third case lacks a detailed description but was diagnosed as an anaplastic thyroid carcinoma [8].

Herein, we present a primary thyroid carcinoma with features of thyrocyte differentiation including colloid production and nuclear features partially emulating papillary thyroid carcinoma. We will describe the cytologic and histologic findings, the immunohistochemical features, and the molecular genetic results. We will compare our patient's case with the three other reported cases of NUTM1 fusion carcinomas arising in the thyroid.

\section{Materials and Methods}

\section{Review of Cytological and Histological Materials}

The cytologic samples were obtained by ultrasound-guided fine-needle aspiration (FNA) of the primary thyroid tumor and intra-operative smears of the resected tumor. The cytopathologist co-authors reviewed the cytology smears and cell block sections. Papanicolaou, DiffQuik ${ }^{\mathrm{TM}}$, and rehydrated hematoxylin and eosin smears were prepared in addition to a formalin-fixed paraffin-embedded (FFPE) cell block of FNA rinses.

The histologic specimen was reviewed by the pathologist co-authors and comprised hematoxylin and eosin-stained sections of tumor blocks and adjacent thyroid parenchyma and neck soft tissue. One pathologist manually counted nuclear grooves, mitotic figures, and individual necrotic cells by quantifying two foci of 500 tumor cells each in histologically viable tumor regions.

\section{Immunohistochemistry Methods}

Immunohistochemical (IHC) staining was performed initially on de-stained Papanicolaou cytology smears and thereafter on two representative tumor sections from the resection specimen. All IHC staining except $\mathrm{BRAF}^{\mathrm{V} 600 \mathrm{e}}$ and NUT antibody staining was performed at the University of Kentucky Health Sciences Center Clinical Laboratory using a Roche automated platform (Ventana, Roche US diagnostic headquarters, Indianaopolis, IN) or Dako automated platform (Autostainer Link 48, Glostrup, Denmark) with validated commercial antibodies using appropriate positive and negative controls. NUT antibody staining was performed by two commercial laboratories, Mayo Clinic Laboratories (Rochester, MN) and ARUP Laboratories (Salt Lake City, UT), and the slides including positive controls were reviewed by the study pathologists. Specific manual counts of percent reactivity for Ki67, p63, and p40 were performed by one study pathologist on 10 consecutive fields of 100 tumor cells each.

\section{Genetic Analysis Methods}

Two dedicated FNA passes were performed and directly rinsed into a Veracyte FNAprotect ${ }^{\circledR}$ collection tube and sent for molecular analysis by the Afirma Xpression Atlas (XA) (Veracyte, South San Francisco, CA). This panel includes coverage of 905 genomic variants and 235 fusion pairs from 593 genes using targeted DNA sequencing and transcriptome RNA sequencing, as previously described. Notably, NUTM1, NSD3, BRD3, and BRD4 fusions are not covered in the XA test (Afirma website, https://www.afirma.com).

FFPE tissue from the thyroidectomy specimen was sent for molecular analysis utilizing the Caris Molecular Intelligence Profile platform (Caris Life Sciences, Phoenix, AZ). This mutational analysis by next-generation sequencing (NGS) was performed on FFPE tissue samples using the Illumina NovaSeq 6000 sequencer (Illumina, Inc., San Diego, CA). Over 700 clinically relevant genes were analyzed with high coverage and a high read-depth; a separate panel was designed to enrich for over 20,000 genes at a lower depth. In addition, gene amplification/deletion was analyzed utilizing a $500-\mathrm{Mb}$ single nucleotide polymorphisms panel (Agilent Technologies, Santa Clara, CA). In summary, this panel is validated to detect variant sequences, copy number alterations, tumor mutational burden (TMB), loss of heterozygosity ( $\mathrm{LOH})$, and microsatellite instability. Gene fusion and transcript variant detection by RNA sequencing were performed on mRNA isolated from FFPE tissue using the Agilent SureSelectXT Low Input Library prep chemistry utilizing the SureSelect Human All Exon V7 bait panel and the Illumina NovaSeq, allowing the detection of transcript variants and novel gene breakpoints.

Fluorescence in situ hybridization was performed by Mayo Clinic Laboratories (Rochester, Minnesota) using a NUTM1 break-apart probe set (15q14; 5'NUTM1, 3'NUTM1) as a validated laboratory developed test. The assay was performed on a representative formalin-fixed, paraffin-embedded tissue section.

\section{Case Description and Results}

\section{Clinical Presentation, Treatment, and Follow-Up}

A 72-year-old woman presented to her health care provider after she noted a new, asymptomatic neck mass. She had a 2-year history of hypothyroidism due to Hashimoto's thyroiditis controlled on $25 \mathrm{mcg}$ of levothyroxine. She denied radiation exposure and reported no family history of thyroid disease or thyroid cancer. On exam, a large visible and palpable, firm, fixed right thyroid nodule was noted. Ultrasound 
imaging of the mass revealed a large $4.5 \times 4.6 \times 4.9-\mathrm{cm}$ solid, hypo- to isoechoic nodule in the right mid-inferior lobe extending into the isthmus causing tracheal deviation to the left. Serologic tests were within normal limits except for elevated thyroglobulin $(42.2 \mathrm{ng} / \mathrm{mL})$ and thyroglobulin antibodies $(66.7 \mathrm{IU} / \mathrm{mL})$. Computed tomography (CT) of the patient's neck (Fig. 1a, b) and chest and a PET CT delineated the known mass without evidence of lymphadenopathy or distant disease.

The patient underwent surgical resection and central node dissection. Intraoperatively, the mass involved the right carotid sheath, but the vessel walls were uninvolved. The tumor was adherent to the anterior inferior trachea. Intraoperative frozen section revealed tumor abutting the entire margin on the thyroid specimen as expected. However, an edge of the remaining perichondrium was negative for tumor at frozen section. Both recurrent laryngeal nerves were identified and preserved intact, and both functioned satisfactorily via vagal stimulation after all dissection. Surgically, gross total resection was achieved. The patient recovered well from surgery and was released from the hospital on the fourth post-operative day. Three weeks after surgery, the patient's serum thyroglobulin level had significantly declined to $2.0 \mathrm{ng} / \mathrm{mL}$ and serum thyroglobulin antibody was also lower at $28.1 \mathrm{IU} / \mathrm{mL}$. The patient is currently undergoing adjuvant radioactive iodine ablation of remnant thyroid tissue.

\section{Fine Needle Aspiration Cytology Findings (Fig. 2a-d)}

Direct smears from the fine needle aspiration biopsy of the thyroid tumor were abundantly cellular and consisted of cohesive clumps of unpolarized tumor cells (Fig. 2a). At higher magnification, the tumor cells appeared relatively monotonous with a moderate $\mathrm{N} / \mathrm{C}$ ratio and round to oval nuclei with a few cells exhibiting mild nuclear contour irregularities (Fig. 2b). Most tumor nuclei contained a single prominent nucleolus. A few tumor nuclei exhibited nuclear grooves (Fig. 2c), and extremely rare nuclear pseudo-inclusions were present (Fig. 2d). Dense globules possibly comprising colloid and scant wispy myxoid material were intermixed within tumor cell groups (Fig. 2c). The background was necrotic to bloody. Two Papanicolaoustained slides were de-stained to perform immunocytochemical staining. Tumor cells diffusely expressed AE1/ AE3 cytokeratins and did not express CD45. A diagnosis of carcinoma was rendered noting a differential of papillary thyroid carcinoma, poorly differentiated thyroid carcinoma, and less likely, metastatic carcinoma.

\section{Resection Findings and Ancillary Testing Results}

Gross Findings (Fig. 3a-d): At resection, the 5-cm tumor involved the right and left lobes and isthmus and extended posteriorly and laterally into surrounding soft tissue. The tumor comprised closely apposed, soft, pink to red nodules with foci of necrosis and hemorrhage.

Microscopic Findings: The tumor replaced $70 \%$ of the thyroid and extended into perithyroidal soft tissue. At low power, the tumor formed solid nodules separated by fibrous septae (Fig. 3a). Within tumor nodules, cells were arranged as vague swirls around peculiar, micro-proliferated blood vessels reminiscent of the abnormal vessels of glioblastoma (Fig. 3b). Ten percent of the tumor exhibited a peritheliomatous growth pattern due to peripheral necrosis. Found in all tumor sections, small, dense eosinophilic globules consistent with colloid were interspersed among tumor cells. These foci were consistent with poorly formed follicles (Fig. 3c). Intracellular colloid was also identified. Wispy gray-blue myxoid matrix was focally present. The squamoid tumor cells were moderate in size (3-4 times the size of a lymphocyte) with a moderate $\mathrm{N} / \mathrm{C}$ ratio, indistinct cell borders, and pale eosinophilic cytoplasm. Most tumor nuclei were oval to round featuring granular chromatin and typically one nucleolus.
Fig. 1 Computerized tomography $(\mathrm{CT})$ of the $4.5 \times 4.5 \times 5.5$ cm thyroid tumor: a sagittal and $\mathbf{b}$ coronal views showing a heterogeneous mass centered in the right lobe of the thyroid and extending into surrounding soft tissue causing mild leftward deviation of the trachea and larynx
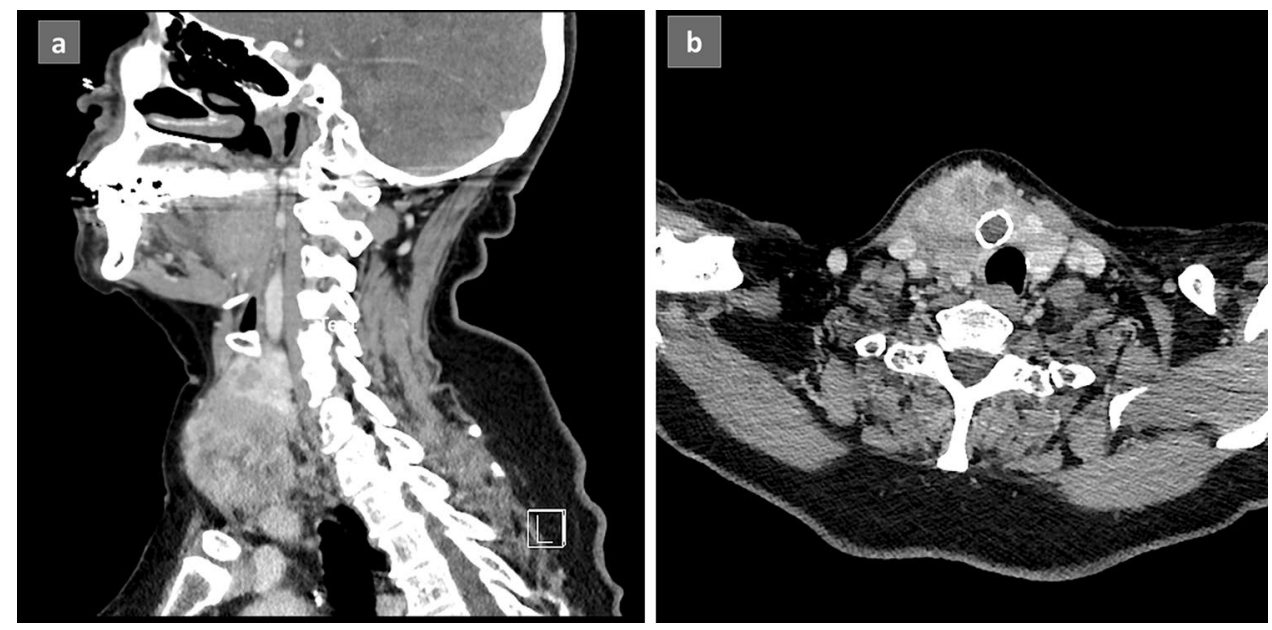

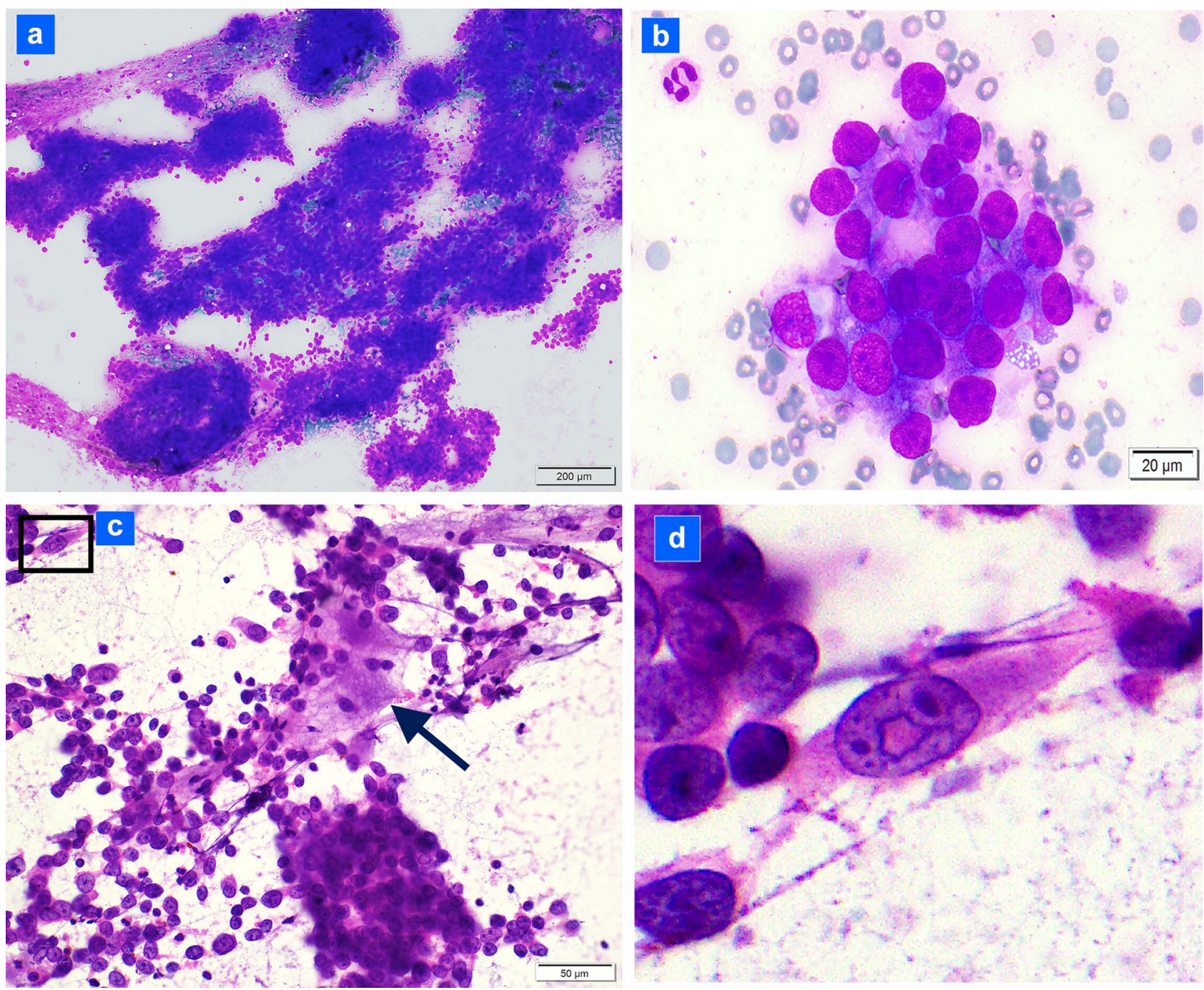

Fig. 2 Cytologic features of the tumor: a on immediate smear, the tumor comprises cohesive groups of disorganized cells (DiffQuik ${ }^{\mathrm{TM}}$; $100 \times$ ); b tumor cells exhibit a moderate to high nuclear to cytoplasmic ratio, round to oval nuclei and size $\sim 3-5$ times that of a red blood cell (DiffQuik ${ }^{\mathrm{TM}}$; $600 \times$ ); c tumor cells exhibit prominent nucleoli

However, interspersed cells exhibited nuclear features suggestive of papillary thyroid carcinoma including irregular nuclear contours, nuclear clearing, and nuclear grooves (up to $7 \%$ of tumor cells) (Fig. 3d). Nuclear pseudo-inclusions were not identified. Mitoses averaged 8 per 10/hpf (up to 24 per 10/hpf). Individual necrotic cells were present averaging 1-2/hpf. Overt squamous differentiation, true papillae, and well-formed follicles were absent. Vascular invasion was present in one medium-sized vein. The residual noninvolved thyroid showed multinodular hyperplasia, mild chronic lymphocytic thyroiditis, and one, $0.8-\mathrm{cm}$, acellular calcified nodule. A well-differentiated component such as papillary thyroid carcinoma or follicular carcinoma was not identified. Nine level VI lymph nodes were negative for carcinoma. The tumor was staged as pT3a/pN0 (following the with focal myxoid matrix (arrow) and rare nuclear pseudo-inclusion (box) (rehydrated hematoxylin and eosin immediate smear; 400×); d higher magnification of the tumor cell with a nuclear pseudo-inclusion (same cell seen in upper left of Fig. 2c; hematoxylin and eosin immediate smear; $600 \times$ )

AJCC Cancer Staging Manual, 8th ed). After review of the immunostaining panel results, and in light of the negative Afirma XPression Atlas ${ }^{\mathrm{TM}}$ results (see below), a diagnosis of "poorly differentiated thyroid carcinoma" was conferred along with a strong recommendation for broad panel nextgeneration sequencing testing due to the unusual histologic features and lack of typical molecular abnormalities.

\section{Immunohistochemistry Results (Table 1; Fig. 4a-f)}

\section{Positive}

Tumor cells diffusely expressed monoclonal PAX8 (Fig. 4a), TTF1 (Fig. 4b), and cytokeratin 7 with patchy expression of BCL2. Less than $2 \%$ of cells expressed p63 and p40. 

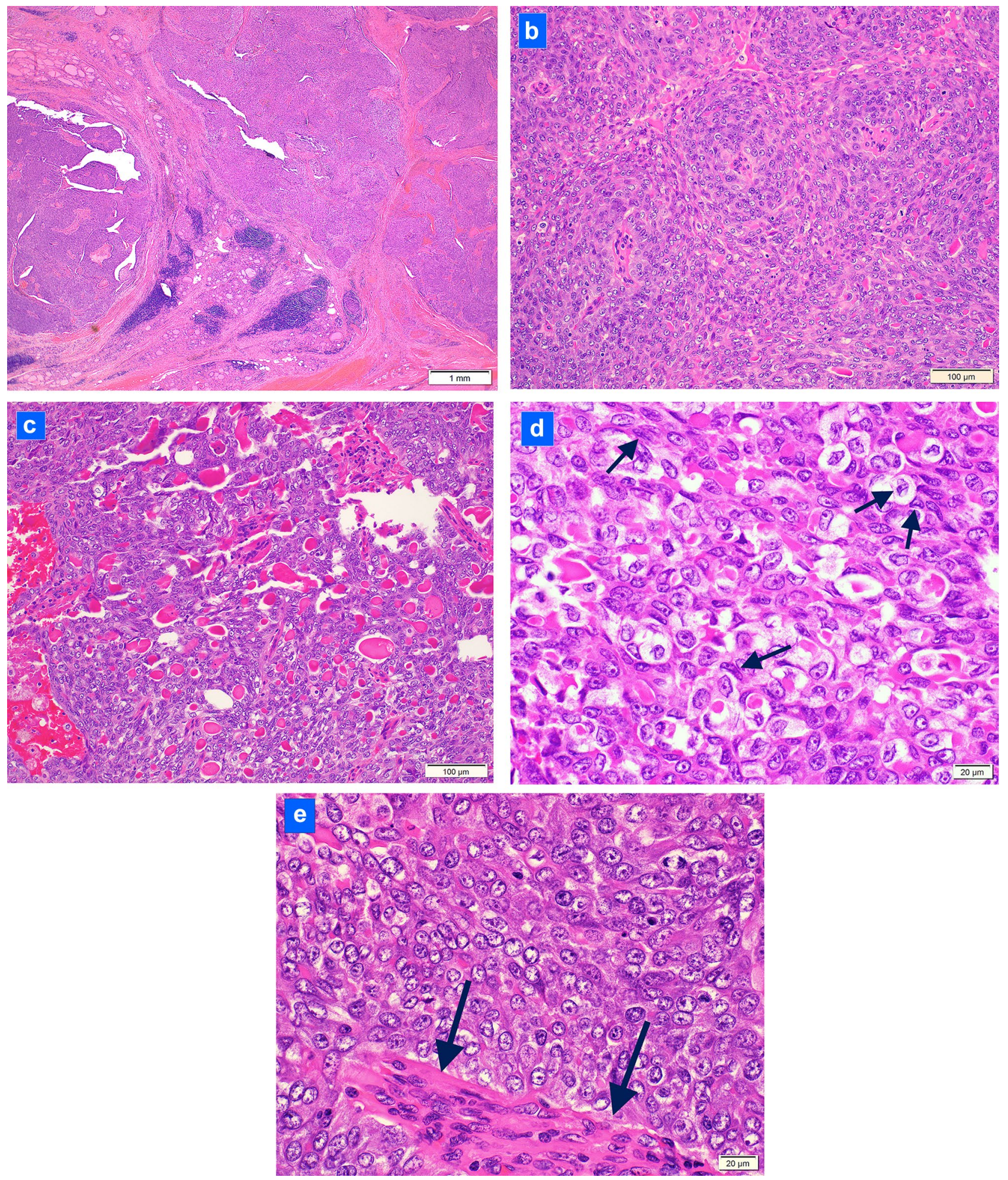

Fig. 3 Histologic features of the tumor at resection: a the tumor forms multiple nodules in the thyroid (hematoxylin and eosin; $40 \times$ ); b most of the tumor comprises nodules of tumor cells swirling around peculiar, distinctive, micro-proliferated blood vessels (hematoxylin and eosin; 200×); c numerous colloid globules intermixed among tumor cells (hematoxylin and eosin; 200×); d tumor cells exhibiting irreg- ular nuclear contours, nuclear clearing and nuclear grooves (arrows) reminiscent of papillary thyroid carcinoma (hematoxylin and eosin; $600 \times$ ); e tumor cells with nuclear clearing but no nuclear grooves and smooth nuclear contours and abnormal, micro-proliferated blood vessel (arrows) (hematoxylin and eosin; 600 $\times$ ) 
Table 1 Antibody details with staining results

\begin{tabular}{|c|c|c|c|}
\hline Antibody & Result & Clone & Manufacturer \\
\hline Cytokeratins AE1/AE3 & Diffusely positive & AE1/AE3 & DAKO \\
\hline Cytokeratin 7 & Diffusely positive & OV-TL $12 / 30$ & DAKO \\
\hline Cytokeratins $5 / 6$ & Negative & D5/16 B4 & DAKO \\
\hline p63 & Negative $(<2 \%)$ & $4 \mathrm{~A} 4$ & Biocare \\
\hline $\mathrm{p} 40$ & Negative $(<2 \%)$ & BC28 & Biocare \\
\hline NUT & Equivocal: $<10 \%$ staining & Unknown & $\begin{array}{l}\text { Unknown (performed at } \\
\text { two external reference } \\
\text { labs) }\end{array}$ \\
\hline e-cadherin & Diffusely positive in tumor cell membranes & $\mathrm{NCH}-38$ & DAKO \\
\hline PAX8 & Diffusely positive & MRQ-50 & Cell Marque/Ventana \\
\hline TTF1 & Diffusely positive & $8 \mathrm{G} 7 \mathrm{G} 3 / 1$ & DAKO \\
\hline Thyroglobulin & $\begin{array}{l}\text { Positive in extracellular colloid and scattered } \\
\text { tumor cells }\end{array}$ & Polyclonal Code IR509 & DAKO \\
\hline BRAF $^{\mathrm{V} 600 \mathrm{E}}$ & Negative & VE1 & Ventana \\
\hline BCL2 & Patchy positive & 124 & DAKO \\
\hline Ki67 & $30-53 \%$ positive & MIB-1 & DAKO \\
\hline $\mathrm{S} 100$ protein & Negative & Polyclonal Code IR504 & DAKO \\
\hline Mammaglobin & Negative & 304-1A5 & DAKO \\
\hline CEA & Negative & IL-7 & DAKO \\
\hline Calcitonin & Negative & Polyclonal Code IR515 & DAKO \\
\hline Chromogranin & Negative & LK2H10 & Cell Marque \\
\hline CD5 & Negative & $4 \mathrm{C} 7$ & DAKO \\
\hline CD45 & Negative & $2 \mathrm{~B} 11+\mathrm{PD} 7 / 26$ & DAKO \\
\hline
\end{tabular}

Thyroglobulin uniformly highlighted intra-tumoral interspersed eosinophilic globules confirming colloid composition (Fig. 4c). Dual IHC staining with e-cadherin and thyroglobulin also revealed scattered tumor cells containing intracellular, cytoplasmic thyroglobulin (Fig. 4d). Weak focal expression of NUT antibody in $10 \%$ of tumor cells (tested twice with antibodies from two commercial laboratories) was interpreted as equivocal (Fig. 4e, f). The proliferation index measured by Ki67 expression ranged from 30 to $53 \%$ (from manual counts of cold and hot spots of reactivity, respectively).

\section{Negative}

There was no expression of $\mathrm{BRAF}^{\mathrm{V} 600 \mathrm{e}}$ protein, cytokeratins 5/6, calcitonin, chromogranin, CEA, CD5, S-100 protein, and mammaglobin. Table 1 lists the antibodies, clone, manufacturer, and results.

\section{Genetic Testing}

\section{Afirma Xpression Atlas ${ }^{\mathrm{TM}}$ Results}

The Afirma XA test detected no gene variants or fusions.

\section{CARIS MI Profile ${ }^{\mathrm{TM}}$ Results}

NGS sequencing revealed no pathogenic variant mutations in $B R A F, N R A S, R E T, T P 53$, or any other relevant cancer genes. The tumor was microsatellite stable, displayed a low TMB (1), and contained a low level of genomic LOH (7\% of tested genomic segments exhibited LOH; assay threshold $\geq 16$ ). RNA sequencing showed an NSD3::NUTM1 gene fusion at exon 7: exon 3 splice site (transcript ID NM_017778.2/NM_175741.2). Fusions for $B R A F, N T R K 1 / 2 / 3, R E T$, and other relevant genes were not detected.

\section{Mayo Clinic NUTM1 FISH Test Result}

The NUTMI break-apart probe FISH test was positive for rearrangement of the NUTM1 locus (Fig. 5). (FISH was performed as an alternate confirmatory method of the NGS results.)

\section{Discussion}

This case is the first reported of a thyroid carcinoma exhibiting thyrocyte differentiation and an NSD $3:$ NUTM1 fusion. 

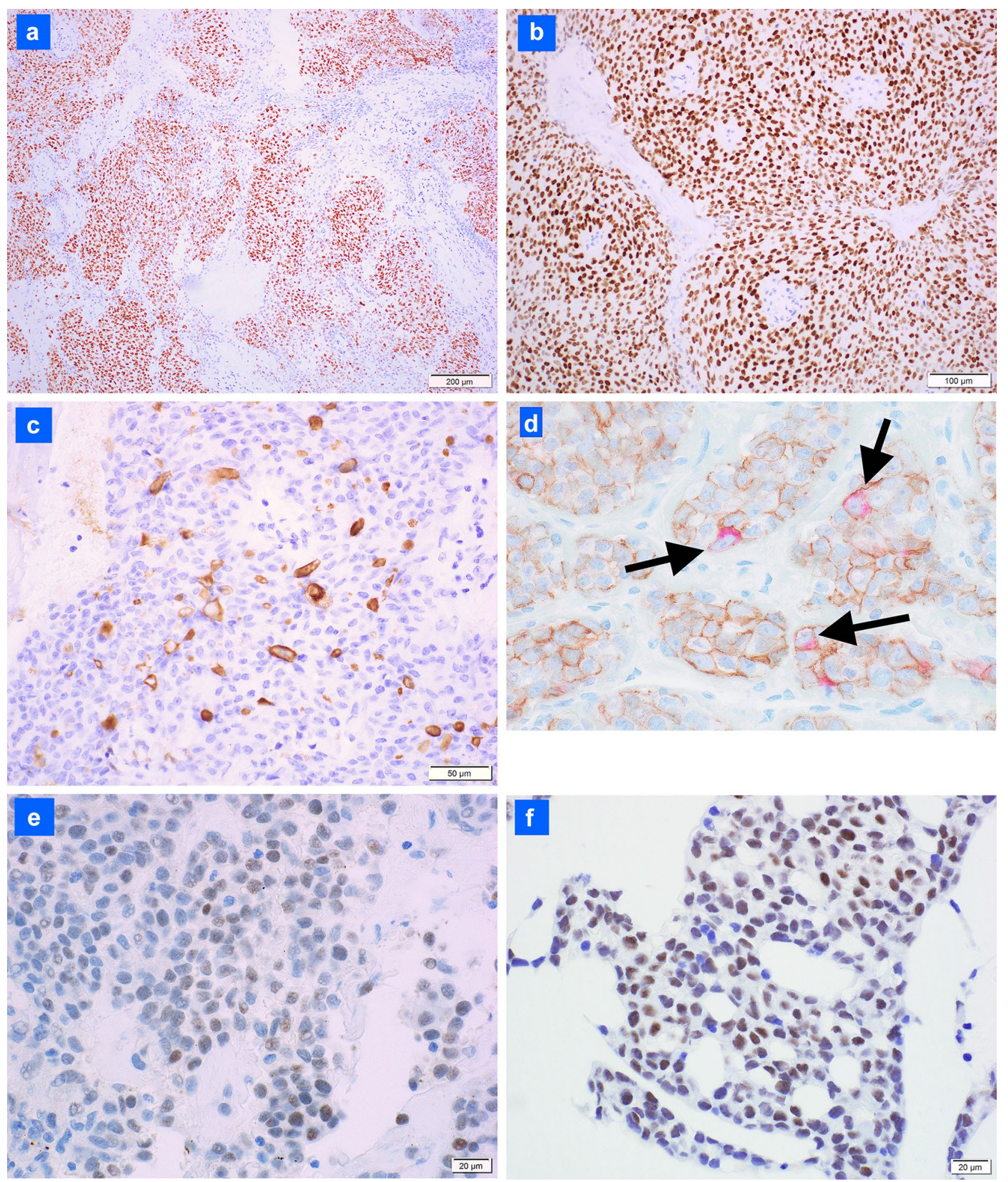

Fig. 4 Selected immunohistochemical results (a brown chromogen is used for all antibodies with hematoxylin as a counter-stain except Fig. $4 \mathrm{~d}$ in which a red chromogen is also used): a PAX8 is expressed in most tumor cells $(100 \times)$; b TTF1 is strongly diffusely expressed $(200 \times)$; $\mathbf{c}$ thyroglobulin antibody strongly decorates colloid globules $(400 \times)$; d intracellular thyroglobulin is present in scattered tumor

cells: e-cadherin (brown chromogen) outlines the cell membrane and surrounds thyroglobulin (red chromogen) (arrows; 600×); e focal ( $10 \%$ of tumor) equivocal weak NUT protein expression (Mayo Clinic Laboratories antibody; $600 \times)$; f focal (10\% of tumor) NUT protein expression (ARUP Laboratories antibody; $600 \times$ ) 


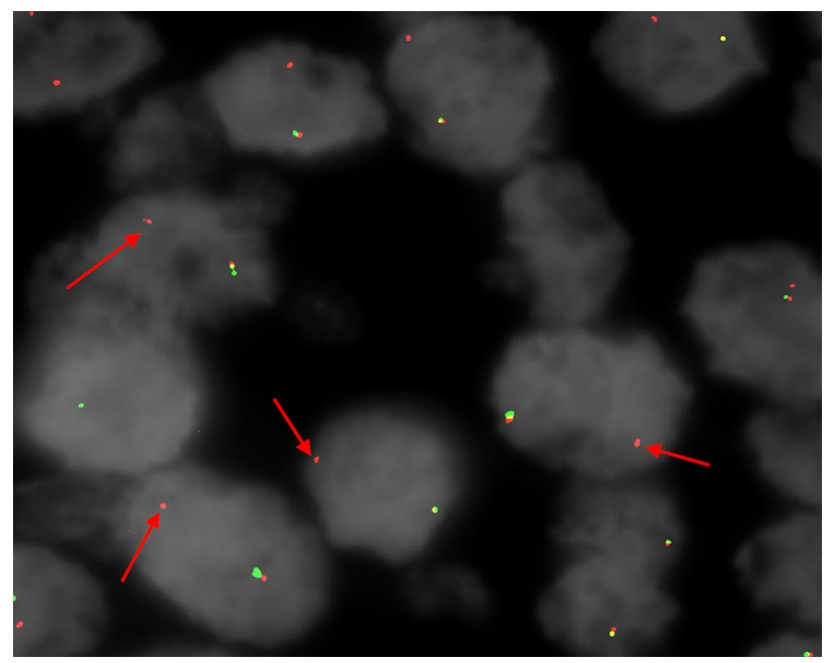

Fig. 5 NUTM1 break-apart fluorescence in situ hybridization (FISH) result showing loss of $5^{\prime} N U T M 1$ signal (green fluorophore) and retention of $3^{\prime} N U T M 1$ signal (red fluorophore, arrows) consistent with rearrangement (Mayo Clinic Laboratories NUTM1 FISH assay; $\sim 600 \times)$

In contrast to all other reported cases of NUT carcinomas harboring a NUTM1::bromodomain complex gene fusion, our patient's tumor showed histologic and immunophenotypic features of a specific, non-squamous carcinoma: (1) thyrocyte differentiation with prominent colloid production, (2) diffuse expression of PAX8 (monoclonal) and TTF1 with thyroglobulin reactivity in colloid, and (3) essentially absent ( 0 to $<2 \%$ ) expression of p63, p40, and cytokeratins 5/6. The unequivocal thyroid features by histology and immunophenotype suggest this carcinoma is better classified as a highgrade thyroid carcinoma than a variant NUT carcinoma.

Identification of this histologically unusual tumor as a NUTM1 fusion thyroid carcinoma required recognition of the histology as unusual, perfoming a panel of IHC antibody stains, and following up initial negative genetic test results with extended molecular genetic testing. We pursued additional, expanded molecular testing on the resected tumor because of the puzzling absence of specific thyroid carcinoma molecular alterations in the Afirma Xpression Atlas ${ }^{\mathrm{TM}}$ test performed on the preceding cytology specimen. Importantly, some commercial molecular genetic panels that are configured specifically for the identification or exclusion of thyroid malignancy do not contain NUTM1 fusions, including the Afirma Xpression Atlas ${ }^{\mathrm{TM}}$ assay (refer to the Afirma website for the list of alterations covered). In a case of a thyroid carcinoma with unusual morphology and absence of $B R A F^{\mathrm{V} 600 \mathrm{E}}$ and $R A S$ mutations (assessed by IHC or targeted genetic testing), a broader NGS panel that includes capacity to detect NUTM1 fusions may be considered in the workup of the tumor.

NUT carcinoma is mechanistically driven by the oncogenic activity of the fused NUT protein and a bromodomain complex (BDC) protein [9]. Histologically, these NUTM1::bromodomain complex gene fusion tumors comprise a poorly differentiated or undifferentiated squamous carcinoma (SC), and approximately $33 \%$ exhibit mature squamous differentiation described as commonly "abrupt" [10]. Very few NUT carcinomas exhibit histology discordance from poorly differentiated to undifferentiated SC. These cases with non-classical histology include tumors that exhibit strands of cells embedded in a chondromyxoid matrix resembling myoepithelial carcinoma, basaloid tumors with mucin production and cribriforming pattern akin to basaloid SC, and tumors composed of rhabdoid cells or small round cells [3, 5, 11]. Importantly, for these reported non-classical cases, the tumors usually demonstrated an immunophenotype classic for NUT carcinoma: diffuse p63 and or p40 expression and speckled nuclear expression of NUT antibody in greater than $50 \%$ of tumor cells [3, 5]. However, recently, two primary lung BRD4::NUTM1 fusion carcinomas were reported that showed dual histology with areas typical of NUT carcinoma as well as foci of micropapillary carcinoma. Both cases diffusely expressed p63 and NUT consistent with classic NUT carcinoma, but one case also showed patchy cytokeratin 7, Napsin-A, and TTF1 expression in the micropapillary component, consistent with some divergent adenocarcinoma differentiation [12].

Cytologically, our patient's tumor comprised cohesive groups distinguishing it from other reports of cytologic features of NUT carcinoma which emphasize cellular dyscohesion [13-15]. Nuclear grooves, as found in our case, have been described in one cytology report of a lung BRD3::NUTM1 fusion tumor, but nuclear pseudo-inclusions observed in our case on the cytology specimen have not been reported in NUT carcinomas [15]. Moreover, the scant viscous colloid globules found on cytology smears in our case are unique. Squamous differentiation was absent in our case but has been described in some NUT carcinoma cytology specimens [13, 14]. Evidence of squamous differentiation in these other cases comprised dyskeratotic cells, keratin, and squamous pearls $[13,14]$.

Histologically, at initial diagnostic evaluation, the closest conventional fit for this tumor was poorly differentiated thyroid carcinoma (PDTC). Like PDTC, the tumor exhibited increased mitotic activity and necrosis consistent with the Memorial Sloan Kettering Cancer Center criteria for PDTC. It also conformed to the Turin criteria for PDTC with a solid growth pattern, as well as the increased mitoses and necrosis. However, three features of the tumor were diffusely found and distinctively odd for PDTC: (1) perivascular swirling arrangement of tumor cells around peculiar, microproliferated vessels (vessels such as seen in glioblastoma); (2) abundant colloid distributed predominantly as extracellular globules consistent with poorly formed follicles; and (3) squamoid appearance of most tumor cells but with sparse, 
intermingled cells showing nuclear features suggestive of papillary thyroid carcinoma. PDTC is often associated with a well-differentiated carcinoma such as papillary or follicular carcinoma, but such a component was absent in our patient's tumor. Other diagnoses were also entertained. Anaplastic thyroid carcinoma was diagnostically excluded because of the colloid production, only mild nuclear pleomorphism, and diffuse TTF1 and PAX8 reactivity. Secretory carcinoma was considered due to the droplet-like distribution of the eosinophilic globules, but the immunophenotype, including thyroglobulin expression in the globules, excluded the diagnosis. Cribriform morular carcinoma also entered the diagnostic differential because of the distinct nodularity of the tumor, but the tumor lacked a cribriform architecture and contained colloid which is typically absent in cribriform morular carcinoma.

While the patient's tumor showed a degree of cytologic atypia and mitotic activity compatible with but not sufficient in themselves for a diagnosis of PDTC, the molecular genetic findings also distinguished it from most cases of poorly differentiated thyroid carcinoma. Approximately $85 \%$ of PDTC are postulated to arise from a well-differentiated thyroid carcinoma that has accrued additional genetic alterations in a stepwise molecular progression $[8,16]$. These $85 \%$ of poorly differentiated thyroid carcinomas not only contain $B R A F^{V 600 E}$ or $R A S$ mutations as found in well-differentiated carcinomas but also show additional mutations in the TERT promoter, TP53, PIK3CA pathway genes, and/or others [8, 16-20]. However, up to $14 \%$ of PDTC contain gene fusions (involving RET, ALK, and PPARG), but no NUTMI fusion has been described $[8,16,17]$.

Three other cases of NUT carcinomas arising in the thyroid have been reported (see Table 2) [6-8]. Two cases were diagnosed as NUT carcinoma and one as anaplastic thyroid carcinoma. In distinct contrast to our case, two cases showed the classic histology of NUT carcinoma [6, 7], while the third case lacks evaluable histologic details and was diagnosed as anaplastic thyroid carcinoma [8]. The case lacking histologic details occurred in a 34-year-old woman and was included in a molecular genetic study of poorly differentiated and anaplastic thyroid carcinomas $(n=84$ and 33 , respectively) [8]. No details were provided of either the histologic features or immunophenotype (see Table 2, case 3 ). The first case arose in a 42-year-old woman and exhibited classic features of NUT carcinoma including monomorphic cells with abrupt squamous differentiation (see Table 2, case 1) [6]. Tumor cells diffusely expressed NUT protein and p63. The second case occurred in a 34-year-old man (see Table 2, case 2) [7]. The post-chemotherapy resected tumor comprised a pleomorphic carcinoma with extensive squamous differentiation. Tumor cells expressed TTF1, PAX8, and p63, NUT protein expression was "equivocal," and there was no expression of thyroglobulin. A biopsy that preceded neoadjuvant chemotherapy was described as typical of NUT carcinoma comprising a monomorphic population of cells with abrupt keratinization. Notably, the fusion found in the 2016 reported case was BRD4::NUTM1, while an NSD3::NUTM1 fusion was identified in the other two cases [6-8].

Thus, at the molecular genetic level, three of the now four reported cases of primary thyroid carcinomas with NUTM1 fusions, including our case, contained the NSD3 fusion partner. NSD3 is a histone methyltransferase that binds to the extraterminal (ET) domain of bromodomainextraterminal domain (BET) proteins [21]. The BET proteins, together with NSD3, p300, and others, normally form an epigenetically functional bromodomain complex (BDC) that attaches to specific chromatin sites and acetylates histones allowing transcription to proceed [4]. Oncogenic compound NUT-bromodomain complex proteins, including the NSD3::NUTM1 protein, create a "massive super enhancer" that forces sustained, markedly increased transcription of specific genes including $M Y C$, SOX2, and $p 63$ while repressing transcription of various tumor suppressor genes $[9,13]$. The NSD3::NUTM1 fusion protein functions essentially analogously to the more common BRD4/BRD3::NUTMI fusion protein, but minor differences may be present [5, 22]. Indeed, one minor difference comprises occasional lack of or equivocal NUT protein expression on immunohistochemical staining, which is consistent with what occurred in our case $[5,7]$.

An oncogenic feature of NUT carcinomas also found in some thyroid carcinomas is MYC protein overexpression [4, $23,24]$. Thyroid carcinomas arising through the effects of MYC overexpression include some papillary carcinomas, follicular carcinomas, poorly differentiated carcinomas, and anaplastic carcinomas [25, 26]. In thyroid carcinomas, MYC overexpression develops by various means, including $M Y C$ amplification or rearrangements, $B R A F^{v 600 e}$ mutation interactions, specific long noncoding RNA dysregulation, increased BRD4 protein production, and abnormal TERT expression, among others [25, 27-31]. This patient's tumor may represent another means (e.g., NUTM1 fusion) by which MYC overexpression compels oncogenesis in the neoplastic thyrocyte.

In summary, we present an NSD3::NUTM1 fusion thyroid carcinoma clearly showing thyrocyte differentiation, including colloid production and poorly formed follicles. This tumor may represent a hitherto unrecognized group of NUTM1 fusion-positive, high-grade thyroid carcinomas that exhibit distinctive features of swirling nodular growth of squamoid cells around micro-proliferated blood vessels, prominent colloid production, nuclear features overlapping with papillary thyroid carcinoma in an intermingled subset of tumor cells, and a thyrocyte immunophenotype. The predicted outcome of this carcinoma is unknown; the pT3 


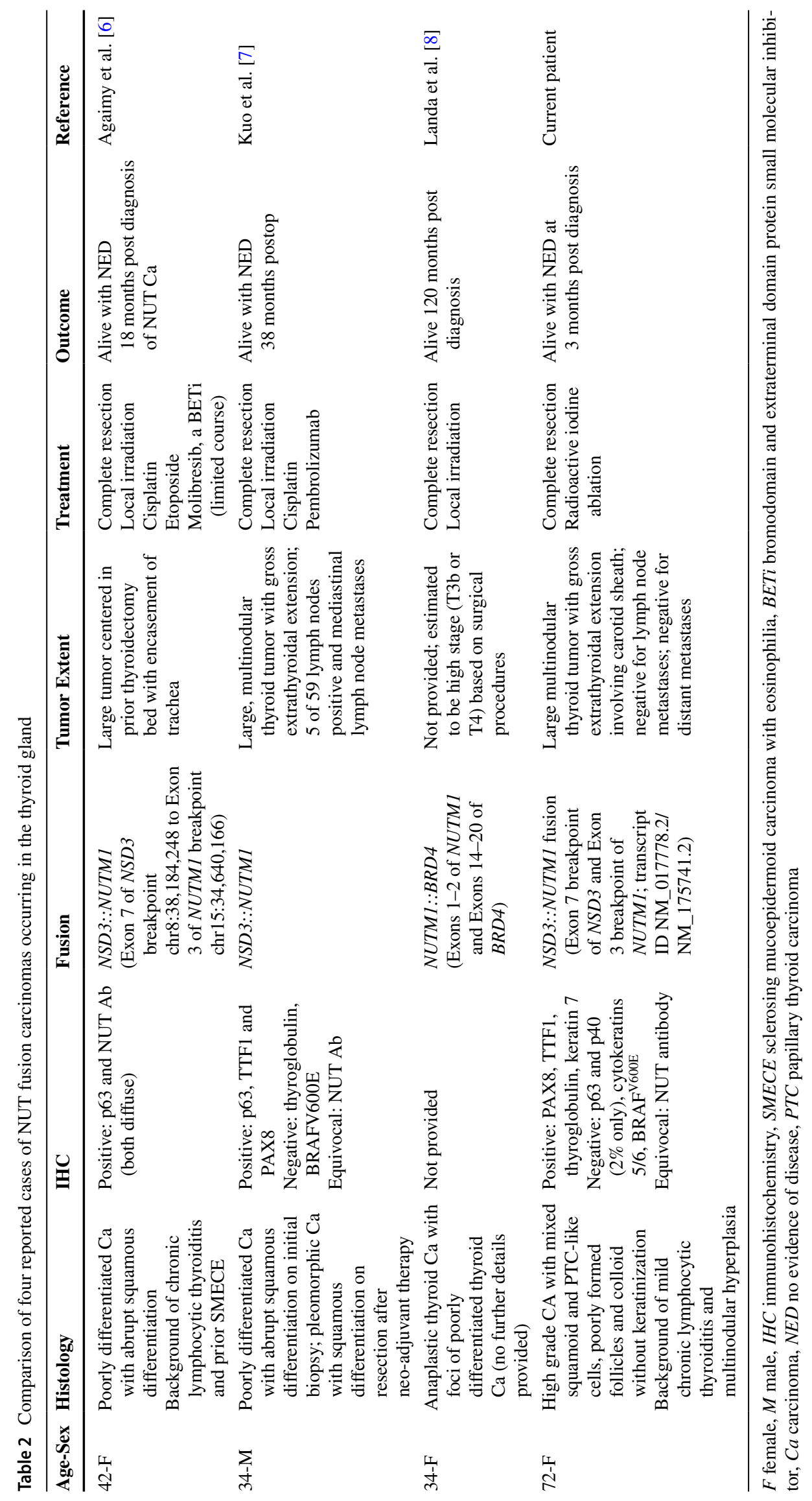


AJCC stage and vascular invasion at presentation suggest it could behave aggressively. Although the three other reported patients with NUTM1 fusion thyroid carcinomas experienced prolonged survival, of the two cases with sufficient details to evaluate, the patients had histologically and/or immunophenotypically typical NUT carcinoma, and moreover, all three were significantly younger than our patient. Should our patient's tumor prove an index case of a specific, high-grade, thyrocyte-differentiated, NUTM1 fusion thyroid carcinoma, discovery of more examples will establish the range of pathology features, tumor behavior, and response to therapies such as BETi agents and immune checkpoint inhibitors.

Acknowledgements The authors thank Dr. Robert B. Jenkins (Mayo Clinic Laboratories, Rochester, Minnesota) for providing a photomicrograph of the NUTM1 fluorescence in situ hybridization test result. The authors also gratefully appreciate the immunohistochemistry expertise of Ms. Monika Ellis.

Author Contribution Manuscript preparation or review: all authors, manual counts on H\&E and IHC stains (JR).

Funding Funding was provided by the University of Kentucky College of Medicine Department of Pathology and Laboratory Medicine.

\section{Availability of Data and Material Available upon request.}

\section{Declarations}

Ethics Approval This observational case report was confirmed as "nonhuman subjects research" by the UK College of Medicine Internal Review Board.

Consent to Participate Not applicable.

Consent for Publication Not applicable.

Conflict of Interest The authors declare no competing interests.

Open Access This article is licensed under a Creative Commons Attribution 4.0 International License, which permits use, sharing, adaptation, distribution and reproduction in any medium or format, as long as you give appropriate credit to the original author(s) and the source, provide a link to the Creative Commons licence, and indicate if changes were made. The images or other third party material in this article are included in the article's Creative Commons licence, unless indicated otherwise in a credit line to the material. If material is not included in the article's Creative Commons licence and your intended use is not permitted by statutory regulation or exceeds the permitted use, you will need to obtain permission directly from the copyright holder. To view a copy of this licence, visit http://creativecommons.org/licenses/by/4.0/.

\section{References}

1. Kubonishi I, Takehara N, Iwata J et al. Novel t(15;19)(q15;p13) chromosome abnormality in a thymic carcinoma. Cancer Research 1991; 51(12): 3327-3328
2. French CA, Miyoshi I, Kubonishi I, Grier HE, Perez-Atayde AR, Fletcher JA. BRD4-NUT fusion oncogene: a novel mechanism in aggressive carcinoma. Cancer Research 2003; 63(2): 304-307

3. Agaimy A, Haller F, Renner A, Niedermeyer J, Hartmann A, French CA. Misleading germ cell phenotype in pulmonary NUT carcinoma harboring the ZNF532-NUTM1 fusion. Am J Surg Pathol. 2021 Jul 8. https://doi.org/10.1097/PAS.0000000000001774. Epub ahead of print. PMID: 34232599

4. French CA. NUT Carcinoma: Clinicopathologic features, pathogenesis, and treatment. Pathol Int. 2018 Nov;68(11):583-595. https://doi.org/10.1111/pin.12727. Epub 2018 Oct 26. PMID: 30362654

5. McEvoy CR, Fox SB, Prall OWJ. Emerging entities in NUTM1rearranged neoplasms. Genes Chromosomes Cancer. 2020 Jun;59(6):375-385. https://doi.org/10.1002/gcc.22838. Epub 2020 Feb 25. PMID: 32060986

6. Agaimy A, Tögel L, Stoehr R, Meidenbauer N, Semrau S, Hartmann A, Mantsopoulos K. NSD3-NUTM1-rearranged carcinoma of the median neck/thyroid bed developing after recent thyroidectomy for sclerosing mucoepidermoid carcinoma with eosinophilia: report of an extraordinary case. Virchows Arch. 2021 Apr 23. https://doi. org/10.1007/s00428-021-03103-8. Epub ahead of print. PMID: 33891143

7. Kuo LE, Barletta J, Schoenfeld JD, White A, French CA, Wong KS, Swanson JR, Lowe AC, et al. NUT carcinoma of the thyroid: an unusual case with a complete response to treatment. Clinical Thyroidology. 2021; 33:38-47. ISSN: 2329-9711

8. Landa I, Ibrahimpasic T, Boucai L, Sinha R, Knauf JA, Shah RH, Dogan S,Ricarte-Filho JC, Krishnamoorthy GP, Xu B, Schultz N, Berger MF, Sander C, Taylor BS, Ghossein R, Ganly I, Fagin JA. Genomic and transcriptomic hallmarks of poorly differentiated and anaplastic thyroid cancers. J Clin Invest. 2016 Mar;126(3):1052-66. https://doi.org/10.1172/JCI85271. Epub 2016 Feb 15. PMID: 26878173; PMCID: PMC4767360

9. Eagen KP, French CA. Supercharging BRD4 with NUT in carcinoma. Oncogene. $2021 \mathrm{Feb}$;0(8):1396-1408. https://doi. org/10.1038/s41388-020-01625-0. Epub 2021 Jan 15. PMID: 33452461; PMCID: PMC7914217

10. French CA. Demystified molecular pathology of NUT midline carcinomas. J Clin Pathol. 2010;63:492-496. https://doi.org/10. 1136/jcp.2007.052902

11. Reddy R, Woods TR, Allan RW, Malhotra P, Mehta HJ, Sarkar PK, Boyce BJ, Asirvatham JR. NUT (Nuclear Protein in Testis) carcinoma: a report of two cases with different histopathologic features. Int J Surg Pathol. 2019 Apr;27(2):225-229. https://doi. org/10.1177/1066896918796606. Epub 2018 Aug 27. PMID: 30149737

12. Chen M, Yang J, Lv L, Li Y, Tang Y, Liu W, Wang W, Jiang L. Comprehensive genetic profiling of six pulmonary nuclear protein in testis carcinomas with a novel micropapillary histological subtype in two cases. Hum Pathol. 2021 Sep;115:5666. https://doi.org/10.1016/j.humpath.2021.02.004. Epub 2021 Mar 10. PMID: 33713695

13. Kuroda S, Suzuki S, Kurita A, Muraki M, Aoshima Y, Tanioka F, Sugimura H. Cytological features of a variant NUT midline carcinoma of the lung harboring the NSD3-NUT fusion gene: a case report and literature review. Case Rep Pathol. 2015;2015:572951. https://doi.org/10.1155/2015/572951. Epub 2015 Jan 19. PMID: 25685583; PMCID: PMC4320876

14. Bishop JA, French CA, Ali SZ. Cytopathologic features of NUT midline carcinoma: a series of 26 specimens from 13 patients. Cancer Cytopathol. 2016 Dec;124(12):901-908. https://doi.org/ 10.1002/cncy.21761. Epub 2016 Jul 11. PMID: 27400194

15. Zhu B, Laskin W, Chen Y, French CA, Cameron MJ, Nayar R, Lin X. NUT midline carcinoma: a neoplasm with diagnostic challenges in cytology. Cytopathology. 2011 Dec;22(6):414-7. 
https://doi.org/10.1111/j.1365-2303.2010.00838.x. Epub 2011 Jan 7. PMID: 21210877

16. Nikiforov Y, Biddinger PW, Thompson Lester DR (2020) Diagnostic pathology and molecular genetics of the thyroid: a comprehensive guide for practicing thyroid pathology, 3rd ed. Wolters Kluwer, Philadelphia

17. Haroon Al Rasheed MR, Xu B. Molecular alterations in thyroid carcinoma. Surg Pathol Clin. 2019 Dec;12(4):921-930. https:// doi.org/10.1016/j.path.2019.08.002. Epub 2019 Sep 27. PMID: 31672298; PMCID: PMC6883923

18. Nylén C, Mechera R, Maréchal-Ross I, Tsang V, Chou A, Gill AJ, Clifton-BlighRJ, Robinson BG, Sywak MS, Sidhu SB, Glover AR. Molecular markers guiding thyroid cancer management. Cancers (Basel). 2020 Aug 4;12(8):2164. https://doi. org/10.3390/cancers12082164. PMID: 32759760; PMCID: PMC7466065

19. Yoo SK, Song YS, Park YJ, Seo JS. Recent improvements in genomic and transcriptomic understanding of anaplastic and poorly differentiated thyroid cancers. Endocrinol Metab (Seoul). 2020 Mar;35(1):44-54. https://doi.org/10.3803/EnM.2020.35.1. 44. PMID: 32207263; PMCID: PMC7090308

20. Duan H, Li Y, Hu P, Gao J, Ying J, Xu W, Zhao D, Wang Z, Ye J, Lizaso A, He Y, Wu H, Liang Z. Mutational profiling of poorly differentiated and anaplastic thyroid carcinoma by the use of targeted next-generation sequencing. Histopathology. 2019 Dec;75(6):890-899. https://doi.org/10.1111/his.13942. Epub 2019 Oct 13 PMID ID: 31230400

21. French CA, Rahman S, Walsh EM, Kühnle S, Grayson AR, Lemieux ME, Grunfeld N, Rubin BP, Antonescu CR, Zhang S, Venkatramani R, Dal Cin P, Howley PM. NSD3-NUT fusion oncoprotein in NUT midline carcinoma: implications for a novel oncogenic mechanism. Cancer Discov. 2014 Aug;4(8):928-41

22. Chau NG, Ma C, Danga K, Al-Sayegh H, Nardi V, Barrette R, Lathan CS, DuBois SG, Haddad RI, Shapiro GI, Sallan SE, Dhar A, Nelson JJ, French CA. An anatomical site and genetic-based prognostic model for patients with nuclear protein in testis (NUT) midline carcinoma: analysis of 124 patients. JNCI Cancer Spectr. 2019 Nov 6;4(2):pkz094. https://doi.org/10.1093/jncics/pkz094. PMID: 32328562; PMCID: PMC7165803

23. Enomoto K, Zhu X, Park S, Zhao L, Zhu YJ, Willingham MC, Qi J, Copland JA, Meltzer P, Cheng SY. Targeting MYC as a therapeutic intervention for anaplastic thyroid cancer. J Clin Endocrinol Metab. 2017 Jul 1;102(7):2268-2280. https://doi.org/10.1210/jc. 2016-3771. PMID: 28368473; PMCID: PMC5505205
24. Lai X, Umbricht CB, Fisher K, Bishop J, Shi Q, Chen S. Identification of novel biomarker and therapeutic target candidates for diagnosis and treatment of follicular carcinoma. J Proteomics. 2017 Aug 23;166:59-67. https://doi.org/10.1016/j.jprot.2017.07. 003. Epub 2017 Jul 12. PMID: 28709933

25. Qu Y, Yang Q, Liu J, Shi B, Ji M, Li G, Hou P. c-Myc is required for BRAFV600E-induced epigenetic silencing by H3K27me3 in tumorigenesis. Theranostics. 2017 May 26;7(7):20922107. https://doi.org/10.7150/thno.19884. PMID: 28656062; PMCID: PMC5485424

26. Sakr HI, Chute DJ, Nasr C, Sturgis CD. cMYC expression in thyroid follicular cell-derived carcinomas: a role in thyroid tumorigenesis. Diagn Pathol. 2017 Oct 3;12(1):71. https://doi. org/10.1186/s13000-017-0661-0. PMID: 28974238; PMCID: PMC5627435

27. Gao X, Wu X, Zhang X, Hua W, Zhang Y, Maimaiti Y, Gao Z, Zhang Y. Inhibition of BRD4 suppresses tumor growth and enhances iodine uptake in thyroid cancer. Biochem Biophys Res Commun. 2016 Jan 15;469(3):679-85. https://doi.org/10.1016/j. bbrc.2015.12.008. Epub 2015 Dec 18. PMID: 26707881

28. Ross J, Miron CE, Plescia J, Laplante P, McBride K, Moitessier N, Möröy T. Targeting MYC: from understanding its biology to drug discovery. Eur J Med Chem. 2021 Mar 5;213:113137. https://doi.org/ 10.1016/j.ejmech.2020.113137. Epub 2020 Dec 29. PMID: 33460833

29. He L, Chen C, Gao G, Xu K, Ma Z. ARV-825-induced BRD4 protein degradation as a therapy for thyroid carcinoma. Aging (Albany NY). 2020 Mar 12;12(5):4547-4557. https://doi.org/ 10.18632/aging.102910. Epub 2020 Mar 12. PMID: 32163373; PMCID: PMC7093165

30. Pestana A, Vinagre J, Sobrinho-Simões M, Soares P. TERT biology and function in cancer: beyond immortalisation. J Mol Endocrinol. 2017 Feb;58(2):R129-R146. https://doi.org/10.1530/ JME-16-0195. Epub 2017 Jan 5. PMID: 28057768

31. Wang Y, Hou Z, Li D. Long noncoding RNA UCA1 promotes anaplastic thyroid cancer cell proliferation via miR-135a-mediated c-myc activation. Mol Med Rep. 2018 Sep;18(3):3068-3076. https:// doi.org/10.3892/mmr.2018.9276. Epub 2018 Jul 16. PMID: 30015867

Publisher's Note Springer Nature remains neutral with regard to jurisdictional claims in published maps and institutional affiliations. 